\title{
A Review: Analysis of PAPR Reduction Techniques of OFDM System
}

\author{
Isha Goel $^{1}$ and Himanshu Monga ${ }^{2 *}$ \\ ${ }^{1}$ Department of Electronics and communication Engineering, India \\ ${ }^{2}$ Jan Nayak Ch. India \\ *Corresponding author: Himanshu Monga, Principal/Director, Sirsa, Haryana, India
}

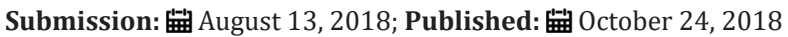

\begin{abstract}
Orthogonal Frequency Division Multiplexing (OFDM) is an efficient multicarrier modulation scheme in wireless communication. It is used in applications like 4G (fourth generation) mobile Communications, wireless networks, digital television, power line networks and audio Broadcasting. It offers both advantages and disadvantages for multicarrier transmission. The main advantages are the elimination of Inter Carrier Interference (ICI) and Inter Symbol Interference (ISI) in the signal. But OFDM's one of the main detrimental aspects are high Peak-to-Average Power Ratio (PAPR) value of the transmitted signal which highly affects the power amplifiers complexity. Several techniques to overcome and reduce PAPR such as Tone Reservation (TR), selective mapping (SLM), clipping and filtering, partial transmit sequence (PTS), etc. are proposed and implemented. Detailed description and comparisons of all techniques are discussed in this paper.
\end{abstract}

Keywords: PAPR; PTS; OFDM; SLM; ICI; ISI; TR; HPA (High Power Amplifier)

\section{Introduction}

With increased demand for high quality communication services in $4 \mathrm{G}$ and $5 \mathrm{G}$, it's been a great challenge to improve the Quality of Service (QoS) and reduces the delay time. To overcome and fulfil this requirement, a technique called Orthogonal Frequency Division Multiplexing (OFDM) is encountered. It over several outfalls like maintaining high data rate, high bandwidth efficiency, low computational complexity and eliminating multipath fading [1].

OFDM is a special case of the frequency division multiplexing (FDM), which is used by the American National Defense Department for military communications. Compared with the conventional FDM, OFDM allows the spectrums from different subcarriers that are orthogonal to be overlapped with each other, which improves the spectral efficiency of the system. The concept of OFDM techniques was first proposed by RW Chang in 1965. In 1967, Saltzberg analyzed the performance of OFDM systems. In 1970, OFDM technology was patented at the USPD and then after it was used in military communication systems. In 1971, SB Weinstern and PM Ebert employed the Discrete Fourier Transform (DFT) into the MCM. in practice, the Fast Fourier Transform (FFT) implementation of the DFT has made OFDM modulation and demodulation feasible and very successful. In the 1980s, ISI was decreased when Peled and Ruiz added cyclic prefix (CP) into the basic OFDM signals to have the orthogonality maintenance among subcarriers [2].
Organization of this paper is in five main sections. Section 2 briefly presents the OFDM system transmitter and receiver side working, its characteristic and the PAPR problem. Literature Review of all the referred past work is mentioned in section 3. In section 4 , the PAPR reduction techniques, their advantages, disadvantages are discussed. Finally, conclusions and future scope are mentioned in section 5 .

\section{OFDM System and PAPR}

\section{OFDM system}

Orthogonal frequency division multiplexing (OFDM) is a most efficient and widely used multiplexing and modulation technology for $4 \mathrm{G}$ and $5 \mathrm{G}$ telecommunications standards which includes digital radio broadcasting, digital terrestrial television (DTT), wireless local area networks (LANs), etc. OFDM systems consist of two parts the transmitter side and the receiver side [3].

\section{OFDM transceiver}

The fundamental principle of OFDM is to split the available bandwidth into multiple sub-carriers. As the number of subcarriers increases, it gets more immune to frequency selective fading, and data rates are also increasing. However, number of sub-carriers cannot be increased arbitrarily because it increases the complex architecture of the system and symbol durations that 
make transmission more sensitive to the time incoherence of the channel.

The problem of the intricate design of the system was handled by Weinstein and Ebert with the implementation of OFDM modulation by Inverse Discrete Fourier Transform (IDFT) and demodulation by Discrete Fourier Transform (DFT). To illustrate this considers one OFDM symbol with $\mathrm{N}$ different subcarriers and assume that $\mathrm{s}(\mathrm{t})$ is sampled at every time interval Ts=N (Figure 1).

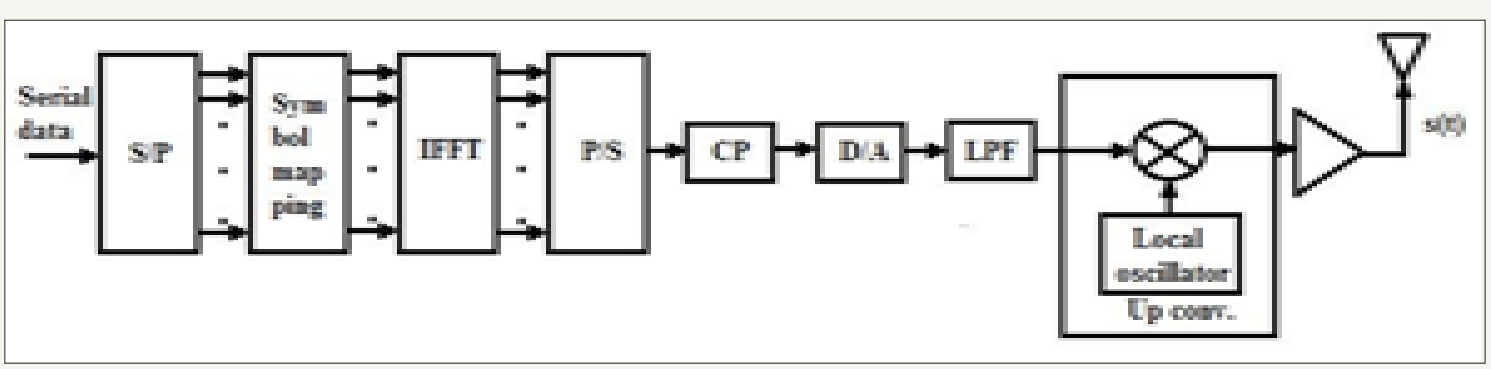

Figure 1: OFDM system transmitter.

\section{OFDM system transmitter}

The incoming serial data is the information that needs to be transmitted through the channel using OFDM system. The serial data is converted into $\mathrm{N}$ different parallel data streams by using serial to parallel converter. These symbols can be modulated by using different modulation techniques and given to the IFFT block as an input [3]. IFFT block gives the digital time domain signal for the given input, and this parallel data is converted into serial data by using parallel to serial converter. The cyclic prefix is introduced between two OFDM symbols to cancel the effect of ISI due to channel dispersion. Now this digital time signal is converted into real time waveform with the use of digital to analog converters. The available baseband signal is up converted to an RF pass band signal with the use of a mixer or modulators.

\section{Channel model}

The phenomenon of noise and multipath environment can be predicted by using channel model. Generation of noise can be done by adding few random data to the OFDM symbol and multipath environment can be generated by adding attenuated and delayed copies of the OFDM signal.

\section{OFDM receiver}

At the receiver end, the received OFDM signal is down converted using the demodulator and sampled with analog-to-digital converters to obtain the digital time domain signal. The digital time domain signal is demodulated by using FFT, and the data that is transmitted can be extracted by using symbol demapper [4] (Figure 2).

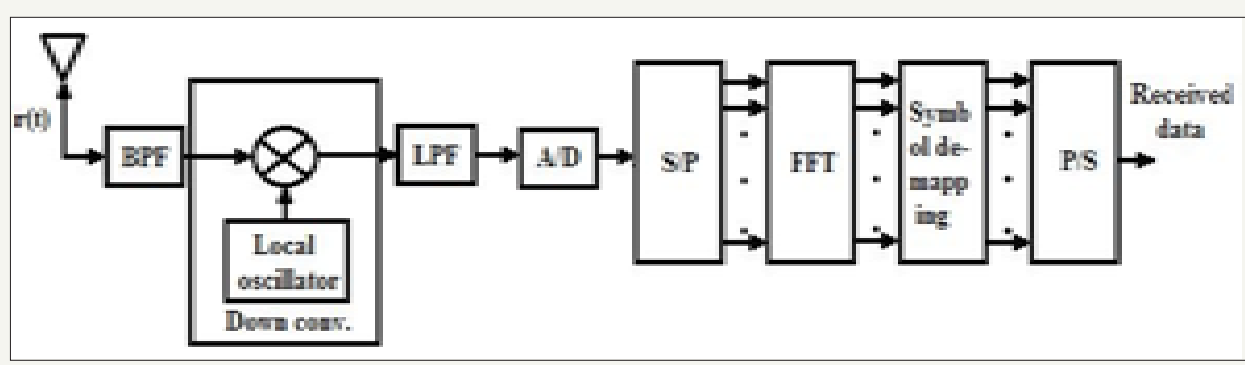

Figure 2: OFDM System receiver.

\section{Characteristics}

OFDM has several features which attract developers to design various standard applications. Even OFDM exhibits numerous advantages over the family of conventional serial modem schemes which are as follows:

A. Implementation complexity: The complexity is significantly lower comparing to the single carrier system for a specified delay spread.

B. Robustness against narrow band interference: An interferer can destroy the communication link in single carrier while a small number of sub carrier get affected in multicarrier.

C. Spectral efficiency: The behavior of orthogonality accommodated a large number of sub carrier in a very narrow spectral region thus increases the spectral efficiency.

D. Immunity against frequency selective fading: Each subcarrier has narrow bandwidth in comparison to overall bandwidth of the signal. It converts a frequency selective fading channel into several nearly flat fading channels. 


\section{PAPR}

The transmitted signal of OFDM exhibits a high peak power when added in the same phase at IFFT. When these signals excite the nonlinear characteristics of the HPA, it causes out-of-band radiation, which further distorts the signals in adjacent bands and its regions. This high peak power reduces the efficiency of HPA and degrades the performance of the OFDM system. To estimate the distortion which is caused by nonlinearity, a well-known parameter used is PAPR.

The PAPR of OFDM continuous time baseband transmitted signal $\mathrm{x}(\mathrm{t})$ is represented as the ratio of maximum instantaneous

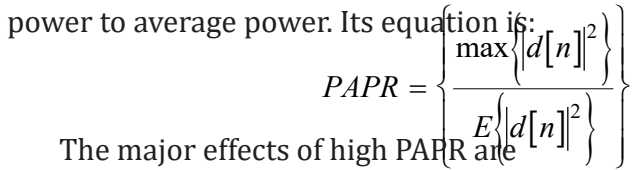

A. Increased complexity in the ADC and DAC.

B. Reduced efficiency of RF (radio frequency) amplifiers [5].

\section{Literature Review}

To the research, different literature and work has been reviewed. Lee \& Kim [6], proposed scheme uses clipping operation to achieve enhanced PAPR reduction of OFDM signals. Since the peak regrowth of signal is mitigated by adaptively modifying the CT, the proposed scheme can effectively enhance the PAPR reduction performance and also reduce the number of iterations. Wang \& Luo [7] investigates. A convex optimization technique to dynamically modify the filter response in an ICF procedure which reduce the PAPR of OFDM symbols. Also, in March 2011 author Jeon [8] used the similarity among the required signals generated in PTS to develop a new algorithm which achieves low computational complexity compared to that of a conventional PTS algorithm.

Chen Ye [9], developed a modified SLM scheme with reduced complexity for decreasing PAPR is proposed. This scheme performs the addition of mapped signal sequences to OFDM signal sequences. Li \& Wang [10], proposed two low-complexity SLM schemes. Although the proposed architectures have certain PAPR performance losses when compared to the traditional SLM scheme but have lower complexities and their BER performances are exactly the same as the traditional SLM scheme.

\section{PAPR Reduction Techniques}

The PAPR reduction includes many techniques, and it's dependent on various factors such as Spectral efficiency, Reduction Capacity, increasing of Transmit signal power, loss in data rate, Computation Complexity, increase in BER, Peak Reduction Carrier. The PAPR reduction techniques have been proposed in order to reduce the PAPR [11] as much as possible. Some of them are:

\section{Clipping and filtering}

1. Coding

2. Partial Transmit Sequence (PTS)
3. Selected Mapping (SLM)

4. Tone Reservation (TR)

5. Tone Injection (TI)

6. Active Constellation Extension (ACE)

7. Companding

\section{Clipping and filtering}

This is the simplest technique used for a PAPR reduction $[12,13]$. In this technique the amplitude of the input signal is frozen to a definite value. Suppose $\mathrm{x}[\mathrm{n}]$ represent the input signal, and the clipped version of $x[n]$ is denoted by $x_{c}[n]$, so expression is defined as:

$$
x_{c}[n]=\left\{\begin{array}{l}
-A x[n] \leq-A \\
x[n]|x[n]|<A \\
A x[n] \geq A
\end{array}\right.
$$

\section{Advantages:}

A. This is a Simple approach.

B. Have high PAPR reduction capability.

C. No side information is required.

D. No change at receiver side.

\section{Drawbacks:}
A. Introduce more distortion.
B. Degrade BER performance.

\section{Coding}

In the coding technique, some code words are used to minimize or reduce the PAPR of the signal. It does not cause any distortion and no out-of-band radiation production, [1] but it has a drawback of reduced bandwidth efficiency as the data rate is reduced. It also suffers from complexity issues, because it requires large memory for finding the best codes and to store large lookup tables, especially for a large number of subcarriers $[1,14]$.

\section{Advantages:}
A. Have less distortion.
B. High PAPR reduction capability.
C. No side information is required.

\section{Drawbacks:}
A. The complexity is very high.
B. Data rate loss.
C. Require modification in both Tx and Rx.

\section{Active constellation extension (ACE)}

In ACE, for reducing PAPR in a particular block, some outer signal constellation points in each block are extended outside of the constellation [15]. 


\section{Advantages:}

A. It is transparent to receiver.

B. There is no loss of data rate.

C. No side information is required.

D. Less distortion of the original signal. Drawbacks:

E. Require addition operations after FFT operation in receiver.

\section{Tone reservation (TR)}

TR works by reserving a few tones within the transmitted bandwidth and assign them appropriate values [14].

\section{Advantages:}

A. No distortion is introduced to the data bearing tones

B. No side information is required.

\section{Drawbacks:}

A. Increase in the average energy per bit, which might reduce the BER performance improvement.

B. Loss of spectral efficiency due to tone reservation.

\section{Selected mapping (SLM)}

In SLM, from the original data block several candidate data blocks are generated and the one with lowest PAPR is transmitted. At the receiver the reverse operation is performed to recover the original data block $[15,16]$.

\section{Advantage:}
A. No distortion is introduced.
B. Independent of number of carriers.

\section{Drawback:}
A. Side information is required.
B. Degrade BER performance.

\section{Partial transmit sequences (PTS)}

The original data block i s partitioned into $\mathrm{V}$ disjoint sub blocks. The subcarriers present in each of these sub blocks are rotated by the equal phase factor, so the PAPR of the combination is minimized. Also, the reverse operation is performed at the receiver, to recover the original data block [17-19].

\section{Advantage:}
A. Less distortion is introduced.
B. Less complex.

\section{Drawbacks:}

A. Side information is required.
B. Degrade BER performance.

C. Involve complex vector sums at Tx.

\section{Companding}

In Companding technique, we enlarge the small signals while compressing the large signals so that the immunity of small signals from noise will increase. This compression is carried out at the transmitter end after the output is taken from IFFT block. Compression of the signal reduces high peaks, so in this way PAPR reduction of input signal takes place [20].

Advantage: Less distortion is introduced.

Drawback: Loss of data rate.

\section{Tone injection (TI)}

The TI technique, basic idea is to increase the constellation size so each point present in the original constellation could be mapped into various equivalent points. In an original constellation point, the equivalent constellation points are added so PAPR reduced. The calculation of the sub-carrier time domain signals also gives lowered PAPR [20].

\section{Advantage: No data rate loss.}

Drawback: Require modulo-D addition, after an FFT operation in the receiver.

\section{Conclusion and Future Scope}

In this paper, we have analyzed and compared eight PAPR reduction techniques. Among above analyzed techniques, it was found out that from present techniques no technique is fully effective in reduction of PAPR and is the best for the OFDM system. As before choosing the appropriate PAPR technique, various other factors like maintaining data rate, computational complexity, BER, signal power should also be taken into acknowledgement. So, it is suggested to propose peak to average power ratio (PAPR) reduction scheme and design a network/model supporting it for OFDM systems after comparing existing conventional methods..

\section{References}

1. http://www.ni.com/en-in/innovations.html

2. Litwin, Louis, Pugel, Michael (2001) The principles of OFDM. RF Signal Processing, pp. 30-48.

3. Yao Y, Ma S, Hu J (2013) A PAPR reduction scheme with residue number system for OFDM. EURASIP Journal on Wireless Communications and Networking, pp. 1-11.

4. Bingiham J (1990) Multicarrier modulation for data transmission: an idea whose time has come. IEEE Commun Mag 28(5): 5-14.

5. Han SH, Lee JH (2005) An overview of peak-to-average power ratio reduction techniques for multicarrier transmission. IEEE Wirel Commun 12(2): 56-65.

6. Lee BM, Kim Y (2013) An adaptive clipping and filtering technique for PAPR reduction of OFDM signals. Circuits, Systems and Signal Processing 32(3): 1335-1349. 
7. Wang YC, Luo ZQ (2011) Optimized Iterative Clipping and Filtering for PAPR Reduction of OFDM Signals. IEEE Trans Commun 59(1): 33-37.

8. Jeon HB, Shin DJ (2011) A low complexity SLM scheme using additive mapping sequences for PAPR reduction of OFDM signals. IEEE Trans Broadcast 57(4): 866-874.

9. Chen Ye (2014) PAPR reduction of OQAM-OFDM signals using segmental PTS scheme with low complexity. IEEE Trans Broadcast 60(1): 141-147.

10. Li CP, Wang SH (2010) Novel low complexity SLM schemes for PAPR reduction in OFDM systems. IEEE Trans Signal processing 58(5): 29162921.

11. Yu P, Jin S (2015) A low complexity tone reservation scheme based on time domain kernel matrix for PAPR reduction in OFDM systems. IEEE Trans Broadcasting 61(4): 710-716.

12. Yasir R, Seshadri M (2013) Peak-to-average power ratio reduction in OFDM schemes: a survey and taxonomy. IEEE Communications Surveys and Tutorials 15(4).

13. Lee BM, Kim Y (2013) An adaptive clipping and filtering technique for PAPR reduction of OFDM signals. Circuits, Systems and Signal Processing 32(3): 1335-1349.
14. Wang YC, Luo ZQ (2011) Optimized iterative clipping and filtering for PAPR reduction of OFDM signals. IEEE Trans Commun 59(1): 33-37.

15. Jeon HB, Shin DJ (2011) A low complexity SLM scheme using additive mapping sequences for PAPR reduction of OFDM signals. IEEE Trans Broadcast 57(4): 866-874.

16. Liang HY (2015) Integrating constellation extension and modified SLM to reduce PAPR of OFDM systems. Wireless Personal Communications 80(2): 709-722.

17. Hill G, Faulkner M, Singh J (2000) Reducing the peak-to-average power ratio in OFDM by cyclically shifting partial transmit sequences. Electronics Letters 36: 560-561.

18. Hou J, Ge J, Jing Li (2011) PAPR reduction of OFDM signals using PTS scheme with low computational complexity. IEEE Trans Broadcast 57(1): 143-148.

19. Ghassemi A, Gulliver TA (2008) Fractional sub-blocking for partial transmit sequence OFDM. IEICE Trans Commun E91(10): 3166-3173.

20. Chen Ye (2014) PAPR reduction of OQAM-OFDM signals using segmental PTS scheme with low complexity. IEEE Trans Broadcast 60(1): 141-147.
Creative Commons Attribution 4.0

International License

For possible submissions Click Here

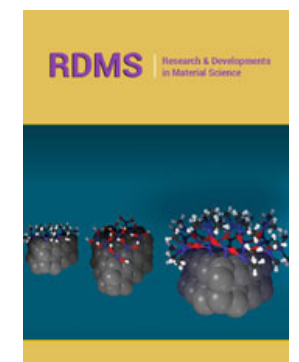

\section{Research \& Development in Material Science}

\section{Benefits of Publishing with us}

- High-level peer review and editorial services

- Freely accessible online immediately upon publication

- Authors retain the copyright to their work

- Licensing it under a Creative Commons license

- Visibility through different online platforms 